\title{
Quantification of sphingosine 1-phosphate by validated LC-MS/MS method revealing strong correlation with apolipoprotein $M$ in plasma but not in serum due to platelet activation during blood coagulation
}

\author{
Cecilia Frej $^{1}$ - Anders Andersson ${ }^{2} \cdot$ Benny Larsson $^{3} \cdot$ Li Jun Guo ${ }^{1} \cdot$ Eva Norström $^{1}$ • \\ Kaisa E. Happonen ${ }^{1} \cdot$ Björn Dahlbäck ${ }^{1}$
}

Received: 13 July 2015 /Revised: 19 August 2015 / Accepted: 25 August 2015 / Published online: 16 September 2015

(C) The Author(s) 2015. This article is published with open access at Springerlink.com

\begin{abstract}
Sphingosine 1-phosphate (S1P) is a signalling sphingolipid affecting multiple cellular functions of vascular and immune systems. It circulates at submicromolar levels bound to HDL-associated apolipoprotein M (apoM) or to albumin. S1P in blood is mainly produced by platelets and erythrocytes, making blood sampling for S1P quantification delicate. Standardisation of sampling is thereby of great importance to obtain robust data. By optimising and characterising the extraction procedure and the LC-MS/MS analysis, we have developed and validated a highly specific and sensitive method for S1P quantification. Blood was collected from healthy individuals $(n=15)$ to evaluate the effects of differential blood sampling on S1P levels. To evaluate correlation between S1P and apoM in different types of plasma and serum, apoM was measured by ELISA. The method showed good accuracy and precision in the range of 0.011 to $0.9 \mu \mathrm{M}$ with less than $0.07 \%$ carryover. We found that the
\end{abstract}

Funding by the Swedish Research Council, the Swedish Heart-Lung Foundation and Söderberg's Foundation.

Electronic supplementary material The online version of this article (doi:10.1007/s00216-015-9008-4) contains supplementary material, which is available to authorized users.

Björn Dahlbäck

bjorn.dahlback@med.lu.se

1 Department of Translational Medicine, Lund University, Skåne University Hospital, Inga Marie Nilssons gata 53, 20502 Malmö, Sweden

2 Department of Clinical Chemistry and Pharmacology, Lund University, Skåne University Hospital, Klinikgatan 19, 22185 Lund, Sweden

3 Department of Clinical Chemistry, Skåne University Hospital, Inga Marie Nilssons gata 53, 20502 Malmö, Sweden methanol precipitation used to extract S1P co-extracted apoM and several other HDL-proteins from plasma. The plateletassociated S1P was released during coagulation, thus increasing the S1P concentration to double in serum as compared to that in plasma. Gel filtration chromatography revealed that the platelet-released S1P was mainly bound to albumin. This explains why the strong correlation between S1P and apoM levels in plasma is lost upon the clotting process and hence not observed in serum. We have developed, characterised and validated an efficient, highly sensitive and specific method for the quantification of S1P in biological material.

Keywords Sphingolipid · Apolipoprotein · Mass spectrometry $\cdot$ Liquid chromatography

\section{Introduction}

Sphingosine 1-phosphate (S1P) is a sphingolipid with pleiotropic functions [1, 2]. Extracellular actions of S1P are mediated via binding and stimulation of five different G-coupled receptors, $\mathrm{S} \mathrm{P}_{1-5}$ [3]. In circulation, $\mathrm{S} 1 \mathrm{P}$ is carried by apolipoprotein $\mathrm{M}(\mathrm{apoM})$ in the lipoproteins and by albumin; approximately $60 \%$ is normally present in high-density lipoproteins (HDL), $10 \%$ in low-density lipoproteins (LDL) and $30 \%$ is bound to albumin [4-6]. S1P is present in plasma in the submicromolar range and can be produced by platelets, erythrocytes and endothelial cells. Platelets store S1P in their plasma membrane and $\alpha$-granules and S1P release requires platelet activation [7]. However, platelets are not the main source of S1P in plasma and thrombocytopenic mice have normal S1P levels [8]. Erythrocytes, which are the most important source of plasma S1P, can phosphorylate sphingosine to generate as well as store S1P but cannot produce S1P de 
novo $[9,10]$. These features make the handling of blood samples taken for S1P analysis important and delicate; however, guidelines for and knowledge on pre-analytical standardisation are limited [11, 12].

Different methods have been used to extract S1P from plasma, e.g. single-phase extraction [13], solid-phase extraction using small gel filtration columns [14], one-step extraction [15-17], two-step extraction [16, 18-22] and methanol precipitation [23-26]. Earlier methods using thin layer chromatography (TLC) showed poor recovery efficiencies and complex sample preparation using radioactive substances $[19,21]$. Later on, high-performance liquid chromatography (HPLC) was introduced to improve automation, separation and resolution. However, S1P is difficult to separate in chromatographic systems because of its zwitterionic properties. Derivatisation with fluorescent molecules like naphthalene2,3-dicarboxaldehyde (NDA) [16] or $o$-phthalaldehyde (OPA) [27] followed by HPLC and detection by fluorescent emission overcome the difficulties with the zwitterionic properties. However, the derivatisation techniques are time consuming and can cause unspecific binding of the fluorescent dye to other targets thereby disturbing the detection of S1P. HPLC coupled to tandem mass spectrometry (LC-MS/MS) provides high resolution and high selectivity and is the most commonly used method for quantifying S1P [23-25, 20, 18, $26,22]$. Due to the complex chemistry of S1P, carryover between sample injections has been identified as a problem. Berdyshev, E.V. et al. reported $10.5 \%$ carryover, which they solved with bisacetylation of the amino group [15]. Other approaches to avoid carryover have been washing the injection needle several times between injections or washing with methanol between each sample analysis [22, 23]. However, most published studies do not address this issue. By optimising the LC system, we have reduced carryover to $0.07 \%$.

We describe a highly sensitive and specific validated method for sample handling and quantification of S1P in plasma and serum using deuterium-marked S1P as internal standard (IS), a quick methanol precipitation and a selective LC-MS/ MS [28] analysis with less than $0.07 \%$ carryover. We demonstrate that apoM correlates with S1P only in platelet-poor plasma (PPP) but not in platelet-rich plasma (PRP) or serum and that S1P released from platelets during blood coagulation mainly binds to albumin and not to apoM.

\section{Materials and method}

\section{Materials}

S1P (D-erythro-sphingosine-1-phosphate), d7S1P (D-erythrosphingosine-d7-1-phosphate) and C17S1P (D-erythro-sphingosine-1-phosphate C17 base) were from Avanti Polar
Lipids (Alabaster, USA). Methanol hypergrade was from Merck (Darmstadt, Germany). Formic acid and essentially fatty acid and globulin-free bovine serum albumin (BSA) were from Sigma-Aldrich (St. Louis, USA). The reversedphase C18 column (XSelect CSH XP C18 $130 \AA, 2.5 \mu \mathrm{m}$, $2.1 \mathrm{~mm} \times 50 \mathrm{~mm}$ ) was from Waters (MA, USA). The 96-well polystyrene plates used for sample injection were from Porvair Sciences (Leatherhead, UK). Tubes for blood collection were from BD, Plymouth, UK (citrate: blue REF 367714, lithium-heparin: green REF 368497, EDTA: purple REF 368499, serum: red REF 367614). The LC-MS/MS system consisted of a Shimadzu Prominence HPLC system CBM20Alite controller with two Shimadzu LC20ADXR pumps, SIL-20AC autosampler, CTO-20AC column-oven and a Rack-changer- $\mathrm{C}$ coupled to a triple quadropole mass spectrometer API 4000 from Sciex (Framingham, USA). Samples were ionised using electrospray ionisation (ESI). Results were calculated by Analyst ${ }^{\mathbb{B}}$ software version 1.6 from AB Sciex (Framingham, USA).

\section{Centrifugation and blood sampling}

Citrate-plasma, lithium-heparin (Li-hep.)-plasma, EDTAplasma and serum were collected from 15 healthy individuals (permission by the local ethical committee at Lund University). Citrate-plasma was centrifuged directly after collection at $300 \mathrm{~g}$ for $15 \mathrm{~min}$ to generate platelet-rich plasma (PRP) and at $1000 \mathrm{~g}$ for $10 \mathrm{~min}, 2000 \mathrm{~g}$ for $10 \mathrm{~min}, 2000 \mathrm{~g}$ for $20 \mathrm{~min}$ and $20,000 \mathrm{~g}$ for $20 \mathrm{~min}$ to obtain platelet-poor plasma (PPP). Serum was left at room temperature for $1 \mathrm{~h}$ to allow the blood to clot. Li-hep-plasma, EDTA-plasma and serum were centrifuged at $2000 \mathrm{~g}$ for $20 \mathrm{~min}$ and $20,000 \mathrm{~g}$ for $20 \mathrm{~min}$ whereafter the samples were stored at $-80^{\circ} \mathrm{C}$ until analysis. Quantification of platelets is described in the Electronic Supplementary Material (ESM). ApoM was quantified as previously described [29].

\section{Stock and working solutions}

S1P was dissolved in methanol to obtain a stock solution of $1 \mathrm{mM}$. Calibration samples (CS) were prepared by diluting the stock in $4 \%$ BSA dissolved in water to obtain concentrations of $0.0037,0.011,0.033,0.1,0.3$ and $0.9 \mu \mathrm{M}$. Aliquots were stored at $-80^{\circ} \mathrm{C}$ and thawed directly before analysis. Samples containing $0.033,0.1$ and $0.9 \mu \mathrm{M} \mathrm{S1P}$ were used as quality controls (QC) in every run. The C17S1P and d7S1P were dissolved in methanol to obtain stock solutions of $200 \mathrm{nM}$ and stored at $-20^{\circ} \mathrm{C}$.

\section{S1P extraction and sample preparation}

Plasma and serum samples $(10 \mu \mathrm{L})$ were diluted with $55 \mu \mathrm{L}$ TBS (50 mM Tris-HCl pH 7.5, 0.15 M NaCl). Precipitation 
solution ( $200 \mu \mathrm{L}$ methanol containing $20 \mathrm{nM}$ IS) was added to $65 \mu \mathrm{L} \mathrm{CS}, \mathrm{QC}$ or the TBS-diluted plasma and vortexed at maximum speed for $30 \mathrm{~s}$. Samples were centrifuged at 17 , $000 \mathrm{~g}$ for $2 \mathrm{~min}$ after which $150 \mu \mathrm{L}$ of supernatants was transferred to a 96 well polystyrene plate, and $5 \mu \mathrm{L}$ was injected for analysis by LC-MS/MS. The protein content of the extract was analysed by running plasma and methanol-extracted plasma on SDS-PAGE gel followed by silver staining and western blotting using an in-house anti-human apoM antibody (rabbit polyclonal anti-human apoM nr 0101), raised against recombinant human apoM (residues 22-188) and characterised as previously described [30, 31], anti-human apoAII (Nordic Biosite, Täby, Sweden), and anti-human apoA1, anti-human apoB100 and anti-human apoE (all from Dako, Glostrup, Denmark).

\section{LC-MS/MS analysis}

Analytes were separated on the reversed-phase C18 column using a gradient of buffers A (water/methanol/formic acid 97/ $2 / 1(v / v / v))$ and B (methanol/acetone/water/formic acid 68/29/ $2 / 1(v / v / v / v))$ with a flow rate of $0.4 \mathrm{~mL} / \mathrm{min}$. The column was maintained at $60{ }^{\circ} \mathrm{C}$ and total time of analysis was $10 \mathrm{~min}$. Ionisation of analytes was made by ESI operating in positive ionisation mode, and the scanning mode was multiple reaction monitoring (MRM). To determine the optimal ion source and MRM parameter settings, S1P and IS were injected postcolumn into the mass spectrometer and full-scan product-ion spectra were obtained (MRM settings are described in Table 1). To monitor the level of contamination of the LC column and mass spectrometer with blood lipids, the three carbon $\mathrm{C}^{13}$ isotopes of palmitoyl-oleoyl-phosphatidylcholine were analysed on $m / z$ transition 763/185. Results were integrated and calculated using linear regression by the Analyst ${ }^{B}$ software. The $10 \%$ dilution factor from the citrate solution in the citrate tubes was compensated for in the calculation of S1P concentration.

Table 1 MRM settings. The specific MRM settings for each ion are presented in the table. The specific tuning parameters for the MS/MS (Sciex API 4000) were ion source temperature $550^{\circ} \mathrm{C}$, ion source voltage $+5500 \mathrm{~V}$, nebulizer gas setting 50 , drying gas setting 60 , curtain gas setting 20 , entrance potential $+10 \mathrm{~V}$ and nitrogen collision chamber gas pressure $6 \mathrm{psi}$

\begin{tabular}{lrlrrr}
\hline Q1 & \multicolumn{1}{c}{ Q3 } & Dwell (ms) & DP & CE & CXP \\
\hline 380.3 & 264.5 & 200 & 81 & 27 & 16 \\
380.3 & 82.1 & 200 & 81 & 45 & 14 \\
387.3 & 271.1 & 200 & 91 & 27 & 14 \\
387.3 & 82.1 & 200 & 91 & 47 & 8 \\
763.6 & 185.3 & 30 & 110 & 43 & 12 \\
\hline
\end{tabular}

\section{Method validation}

Ion suppression test S1P was continuously injected postcolumn to the mass spectrometer to retain a stable S1P signal, and then an ethanol-extracted plasma sample containing the C17S1P was injected pre-column. A countersink of the stabilised S1P signal caused by components in the plasma sample was an indication of ion suppression at that retention time. The ion suppression analysis was made on five individual samples, one of which was serum.

Carryover After analysis of the highest calibration sample $(0.9 \mu \mathrm{M})$, a blank was injected (mobile phase A or TBS buffer) and the S1P peak measured. The degree of carryover was estimated by dividing the S1P peak in the blank with that of the calibration sample from seven individual experiments.

IS purity IS purity was analysed by analysing TBS extracted with methanol containing d7S1P.

Linearity Linearity was analysed by plotting the peak area of the analyte divided with the peak area of IS $(y)$ versus the spiked concentration of S1P $(x)$. The correlation coefficient $\left(\mathrm{R}^{2}\right)$ was calculated as the value of the joint variation between $x$ and $y$.

Recovery Recovery was evaluated by spiking plasma samples with known amounts of S1P and calculated as [(final concentration-initial concentration)/added concentration*100].

Accuracy and precision To evaluate precision and accuracy, QC samples were analysed within runs (intra-run validation) at 10 replicate analyses and between runs (inter-run validation) at 10 different occasions. Accuracy was calculated as the mean of measured concentration/spiked concentration* 100 , and precision was calculated using the coefficient of variation $\mathrm{CV} \%$ as standard deviation/mean of measured concentration* 100 .

Stability and selectivity The methodology for stability and selectivity experiments is described in the ESM.

\section{Gel filtration of serum and plasma}

Citrate-plasma or serum $(500 \mu \mathrm{L})$ pooled from 10 healthy individuals was applied to a Superose 6 10/300 GL column connected to a ÄKTA AVANT (GE Healthcare, Uppsala, Sweden). Gel filtration chromatography was performed in TBS at a flow rate of $0.4 \mathrm{~mL} / \mathrm{min}$, and 30 fractions each containing $300 \mu \mathrm{L}$ were collected. ApoM, S1P and albumin were measured in the fractions using methods described above and with the QuantiChrom BCG Albumin Assay Kit (Bioassay Systems, USA). 


\section{Statistical analysis}

Statistical significance of the differences between groups was measured by Friedman's test with Dun's multiple comparison. Correlation analysis was made according to Spearman. S1P content in gel filtration peaks was measured by analysis of area under the curve. Statistics were calculated using GraphPad Prism 4.0 (GraphPad Software, La Jolla, CA, USA).

\section{Results}

\section{Optimising peak shape and LC column}

S1P contains a hydrophobic C18 aliphatic chain and hydrophilic inorganic phosphoric acid group giving $\mathrm{S} 1 \mathrm{P}$ amphipathic properties. In addition, S1P has a primary amino group and a hydroxyl group bound to the aliphatic chain giving S1P zwitterionic properties. These properties are important to consider when optimising chromatographic separation of S1P since it will affect the intermolecular forces in the LC column. We tested a variety of different LC columns, HILIC and different reversed-phase columns such as $\mathrm{C} 8$ and $\mathrm{C} 18$, but all gave rise to distinct peak tailing, which is an indication of carryover (data not shown). A C18 column from Waters using the Charged Surface Hybrid Technology generated a positively charged surface at low $\mathrm{pH}$. This column together with a buffer system consisting of water-based mobile buffer $\mathrm{A}$ and methanol/acetonebased buffer $\mathrm{B}$, both with a $\mathrm{pH}$ between 1 and 2 , gave a better peak shape without peak tailing and adequate elution time of $3.7 \mathrm{~min}$ (Fig. 1E, ESM Fig. S1A and S1B).

\section{C17S1P versus deuterium-marked S1P as IS}

Initially, we used C17S1P as IS since it is the most commonly used IS for S1P quantification. When analysing methanol extract from human plasma and serum, C17S1P eluted after around $3.4 \mathrm{~min}$ and $\mathrm{S} 1 \mathrm{P}$ after $3.8 \mathrm{~min}$ (Fig. 1A). Since the C17S1P and S1P did not co-elute from the LC column (Fig. 1A), we evaluated for ion suppression at their respective elution timepoints in four individual plasma samples and one serum sample. We observed a similar ion suppression in all analysed samples at the elution time of C17S1P but not at that of S1P (representative chromatogram Fig. 1B-D). Therefore, C17S1P is not an optimal IS as the matrix effect may affect quantification and thereby increase variation. We instead tried deuterium-marked S1P (d7S1P) as IS, which has the advantage that it co-elutes with S1P from the LC system (Fig. 1E, ESM Fig. S1A and S1B). To evaluate IS purity, TBS was extracted with methanol containing IS. No peak at the retention time of S1P larger than noise could be detected (ESM Fig. S1C and S1D).

\section{Validation results}

\section{Linearity and $L L O Q$}

The calibration curve was linear, $R^{2}=0.995(n=10)$, showing excellent correlation between signal $(y)$ and concentration $(x)$ (Fig. 1F). The second lowest CS, 11 nM (Fig. 1G), fulfilled the requirements to be the LLOQ (signal to noise ratio more than 5 and precision and accuracy within 20 and 80-120\%, respectively) (ESM Table S2).

\section{Carryover}

We analysed the carryover effect of S1P by injecting blank (Fig. 1I) after the injection of $0.9 \mu \mathrm{M} \mathrm{CS}$ (Fig. 1H), and the S1P signal in the blank was divided with the signal in the $0.9 \mu \mathrm{M}$ sample. The mean carryover effect from seven individual experiments was $0.065 \pm 0.05 \%$, which can be considered negligible.

\section{Specific HDL-apolipoproteins present in the methanol extract}

When spiking pure methanol with S1P in the submicromolar range, we obtained S1P levels lower than the predicted ones. However, when S1P was added to an albumin-containing solution, the measured values were the same as those predicted (data not shown). Since we use methanol precipitation when extracting S1P from samples, we wanted to investigate if any of the carrier proteins, e.g. apoM or albumin, were coextracted with S1P during the methanol precipitation and

Fig. 1 Evaluation of ion suppression and elution profile for C17S1P, S1P and deuterium-marked S1P. A Elution profile of S1P extracted from human plasma with $\mathrm{C} 17 \mathrm{~S} 1 \mathrm{P}$ as IS. A, B The dark blue and red traces represent the quantification $(\mathrm{m} / \mathrm{z} 380 / 264)$ and qualification ions for S1P (eluted after $3.8 \mathrm{~min}$ ), respectively. The green and grey traces represent the quantification $(\mathrm{m} / \mathrm{z} 366 / 250)$ and the qualification ions $(\mathrm{m} / \mathrm{z} 366 / 82)$ for $\mathrm{C} 17 \mathrm{~S} 1 \mathrm{P}$ (eluted after $3.4 \mathrm{~min}$ ), respectively. The light blue line represents carbon $\mathrm{C}^{13}$ isotopes of palmitoyl-oleoyl-phosphatidylcholine $\left(\mathrm{m} / \mathrm{z}_{\mathrm{z}} 763 / \mathrm{185}\right)$ that elute after 6-7 $\mathrm{min}$. B-D Ion suppression analysis in human plasma. B Elution timepoints for C17S1P, S1P and palmitoyloleoyl-phosphatidylcholine, C quantification ion of S1P $(\mathrm{m} / \mathrm{z} 380 / 264)$ and $\mathbf{D}$ quantification ion for C17S1P $(\mathrm{m} / \mathrm{z} 366 / 250)$. E Elution profile of S1P extracted from human plasma with $\mathrm{d} 7 \mathrm{~S} 1 \mathrm{P}$ as IS. The chromatogram shows ions for S1P and d7S1P both eluting after $3.7 \mathrm{~min}$ and carbon $\mathrm{C}^{13}$ isotopes of palmitoyl-oleoyl-phosphatidylcholine, eluting after 6-7 min. F Plotted standard curve of CS. G S1P signal in $0.011 \mu \mathrm{M}$ CS (LLOQ); H, I carryover evaluation. Representative chromatograms of $0.9 \mu \mathrm{M}$ CS (H) and blank (I) 

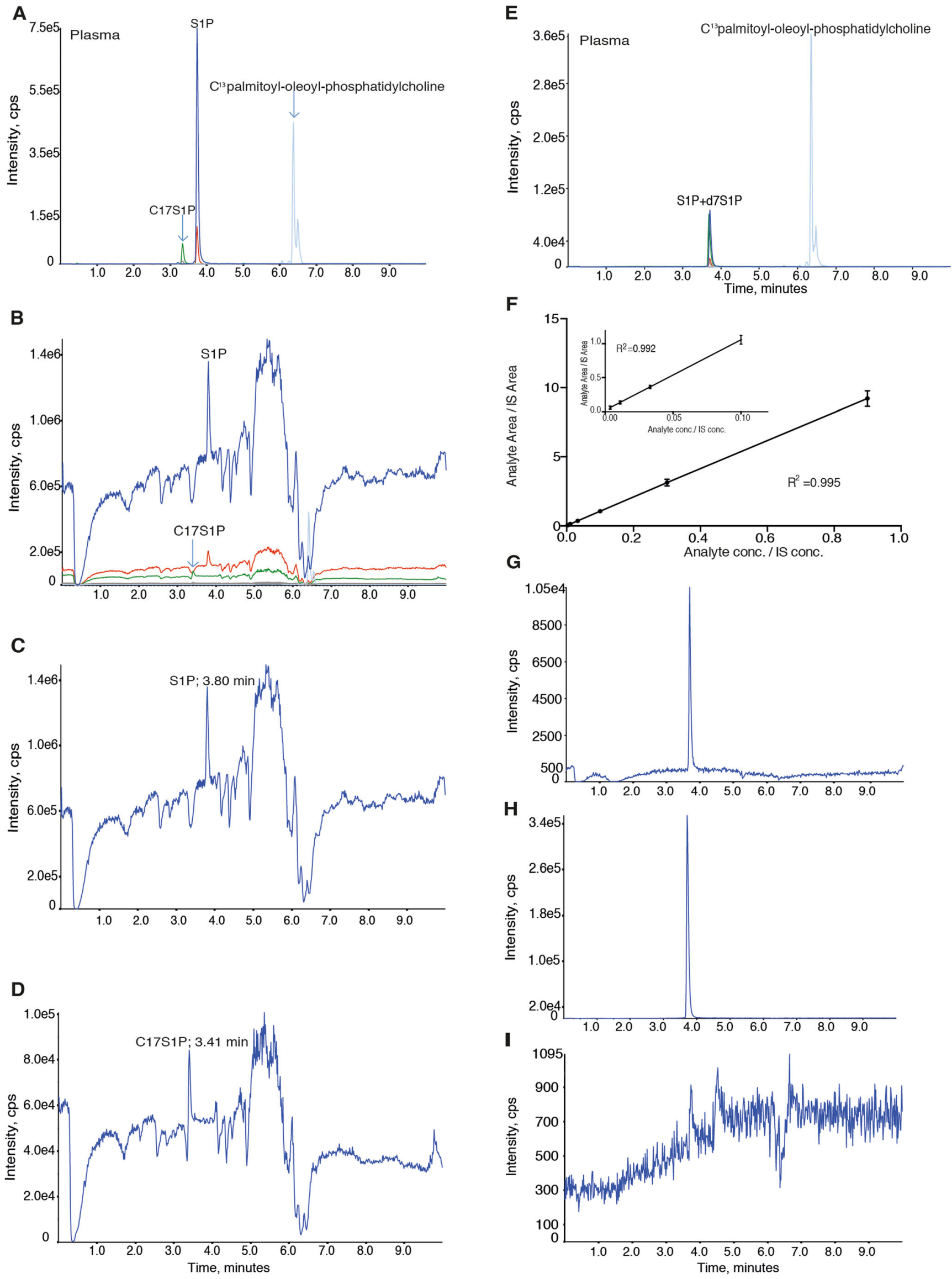
Fig. 2 HDL-proteins in methanol extract. A, B Citrate-plasma was extracted by methanol precipitation and compared to unextracted citrate-plasma. A Total protein analysis by silver staining. B Apolipoprotein content analysed by western blotting

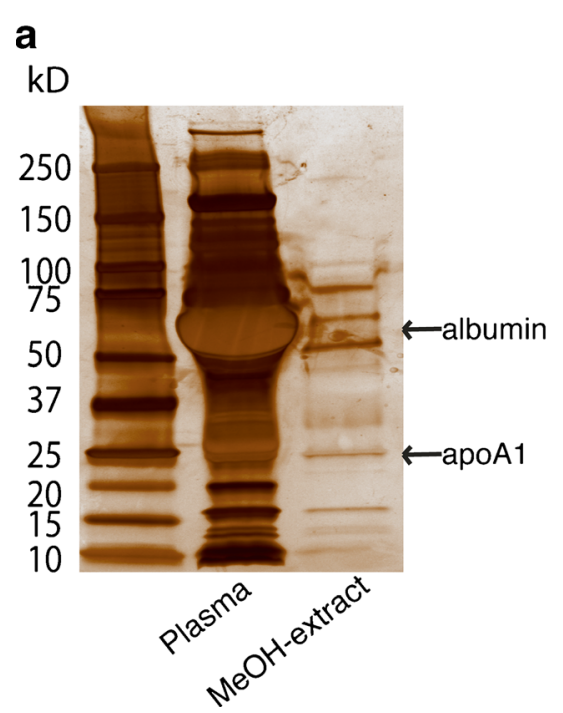

thereby stabilising S1P in the extract. After methanol extraction, both albumin and HDL-proteins were present in the extract as visualised by silver staining and western blotting (Fig. 2A, B). When analysing the presence of apolipoproteins in the extract, we found that the HDL-specific apolipoproteins apoM, apoA1 and apoAII were present in the extract, whereas apoB100 and apoE were not (Fig. 2B).

\section{Selectivity}

When plasma or $0.9 \mu \mathrm{M}$ QC was treated with charcoal, S1P levels decreased by 82.9 and $100 \%$, respectively (ESM Table S1). To further analyse selectivity, HDL and HDL depleted of the S1P carrier apoM (see ESM: Methods) were analysed. The apoM levels dropped by $100 \%$ when apoM was depleted from HDL (ESM Fig. S2A and S2B). The signal for S1P decreased by $93.4 \%$ in the apoM-depleted HDL compared to total HDL (ESM Fig S2A, right panel). Finally, in the selectivity validation, $4 \%$ BSA, TBS and mobile buffer A were analysed. The S1P concentration was very low $(0.5 \mathrm{nM})$ in the $4 \%$ BSA and below detection level in TBS and mobile buffer A (ESM Table S1).

Table 2 Recovery of S1P in spiked plasma samples. Known amounts of S1P were added to citrate-plasma $(n=6)$. Experiments were carried out in duplicate with one analysis for each experiment. Recovery was
Recovery

Recovery was calculated by spiking six plasma samples with known amounts of S1P. Recovery efficiencies varied between 95.2 and $111 \%$ (Table 2). Mean recovery was $103 \%$

Stability of S1P at room temperature and after repeated freeze and thaw cycles

S1P was stable when leaving citrate-plasma, serum and QC samples at room temperature for 0-24 h (ESM Fig S3A-S3C), in agreement with results previously reported for EDTAplasma [17]. When subjecting S1P to repeated freeze and thaw cycles, S1P decreased by $6 \%$ in citrate-plasma after being frozen once but was unaffected by further freeze-thaw cycles (ESM Fig S3D and S3E).

\section{Accuracy and precision}

Values for accuracy and precision are summarised in ESM Table S2. Accuracy and precision for intra- and inter-

calculated as follows [(final concentration-initial concentration)/added concentration*100]

\begin{tabular}{llllll}
\hline Sample no. & Unspiked $(\mu \mathrm{M})$ & Spiked $(\mu \mathrm{M}$, mean $\pm \mathrm{SD})$ & Measured difference $(\mu \mathrm{M})$ & Expected difference $(\mu \mathrm{M})$ & Recovery $(\%)$ \\
\hline 1 & 0.628 & $1.26 \pm 0.009$ & 0.633 & 0.570 & 111 \\
2 & 0.838 & $1.41 \pm 0.028$ & 0.572 & 0.570 & 100 \\
3 & 0.748 & $1.29 \pm 0.060$ & 0.542 & 0.570 & 95.2 \\
4 & 0.510 & $1.11 \pm 0.046$ & 0.602 & 0.570 & 106 \\
5 & 0.696 & $1.28 \pm 0.018$ & 0.585 & 0.570 & 103 \\
6 & 0.838 & $1.42 \pm 0.009$ & 0.585 & 0.570 & 103 \\
\hline
\end{tabular}


Fig. 3 Platelet contamination affecting S1P analysis and the S1P correlation to apoM. Citrateplasma was collected from 15 healthy individuals and centrifuged at different protocols. A Platelet concentration was measured by flow cytometry, B S1P by LC-MS/MS and C apoM by ELISA. D Correlation analysis between S1P and apoM. Results are plotted as individual values. Statistical analysis between groups was made by Friedman's test, and correlation was calculated by Spearman's rank correlation coefficient $\left(r_{\mathrm{S}}\right)$. ${ }^{*} p<0.05 ; * * p<0.01$, $* * * * p<0.0001$
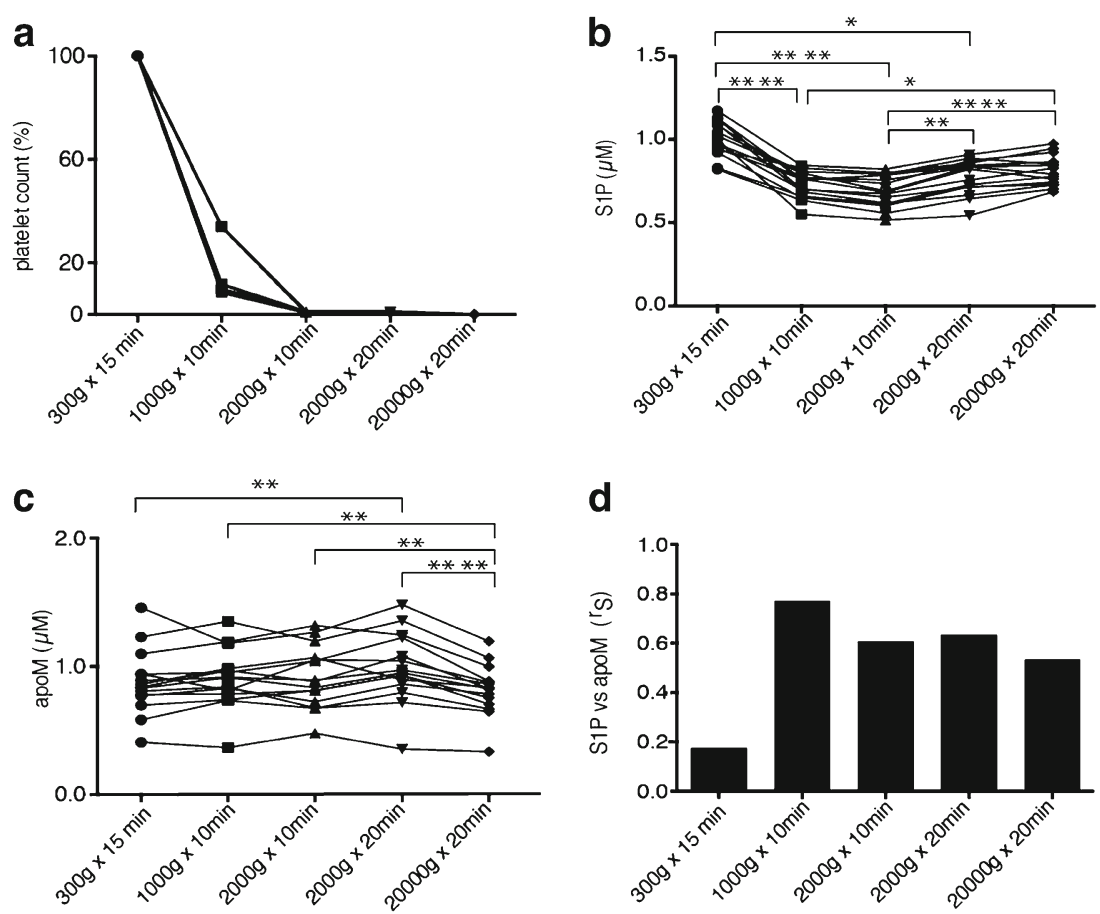

validation were below $12 \%$, which is within recommended limits.

\section{SIP and apoM in platelet-rich and platelet-poor plasma}

To investigate whether different centrifugation protocols leave varying amounts of platelets in the plasma and thus an erroneous high S1P concentration, citrate-plasma was collected from 15 healthy individuals and subjected to different centrifugation protocols. Platelets were measured in the first five individuals to determine how many platelets were left after each centrifugation step (Fig. 3A). S1P was significantly higher in PRP (300g for $15 \mathrm{~min}$ ) compared to PPP $(1000 \mathrm{~g}$ for $10 \mathrm{~min}$, $2000 \mathrm{~g}$ for $10 \mathrm{~min}$ and $2000 \mathrm{~g}$ for $20 \mathrm{~min}$ ) (Fig. 3B). However, there was a noteworthy significant increase of the S1P concentration when the plasma was centrifuged at $2000 \mathrm{~g}$ for $20 \mathrm{~min}$ and more (Fig. 3B). ApoM demonstrated a slight but significant increase in plasma after centrifugation at $2000 \mathrm{~g}$ for $20 \mathrm{~min}$ and a slight decrease after $20,000 \mathrm{~g}$ for $20 \mathrm{~min}$ as compared to plasma that was centrifuged at lower speed and time (Fig. 3C). As the S1P concentration was much higher in PRP compared to PPP and the apoM levels were relatively stable, the correlation between apoM and S1P in PRP was much lower than that observed in PPP (Fig. 3D). Even though there was a slight variation of both apoM and S1P levels between the different centrifugation protocols in PPP (from $1000 \mathrm{~g}$ for $10 \mathrm{~min}$ to $20,000 \mathrm{~g}$ for $20 \mathrm{~min}$ ), the correlation between apoM and S1P between these groups was similar (Fig. 3D).
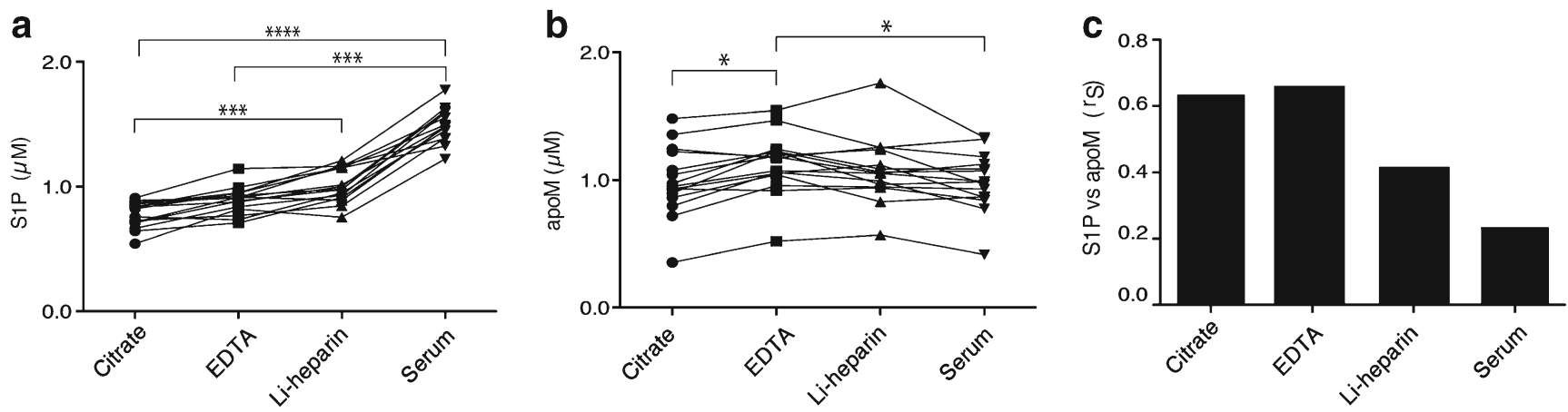

Fig. $4 \mathrm{~S} 1 \mathrm{P}$ and apoM concentrations in different plasma types and in serum. Citrate-, Li-hep- and EDTA-plasma and serum were collected from 15 healthy individuals and centrifuged for $2000 \mathrm{~g}$ in $20 \mathrm{~min}$. A S1P was analysed by LC-MS/MS. B apoM was analysed by ELISA; C correlation analysis between S1P and apoM. Results are plotted as individual values. Statistical analysis between groups was made by Friedman's test, and correlation was calculated by Spearman's rank correlation coefficient $\left(r_{\mathrm{S}}\right) .{ }^{*} p<0.05 ; * * * p<0.001, * * * * p<0.0001$ 
Fig. 5 S1P released during blood coagulation binds to albumin. Citrate-plasma or serum pooled from 10 individuals (total volume $500 \mu \mathrm{L}$ ) was subjected to gel filtration chromatography. S1P was extracted from the collected fractions and analysed in LC-MS/ MS. ApoM was measured by ELISA and albumin was measured by a commercial kit. Area under the curve was calculated to evaluate the S1P content eluted with either apoM or albumin a

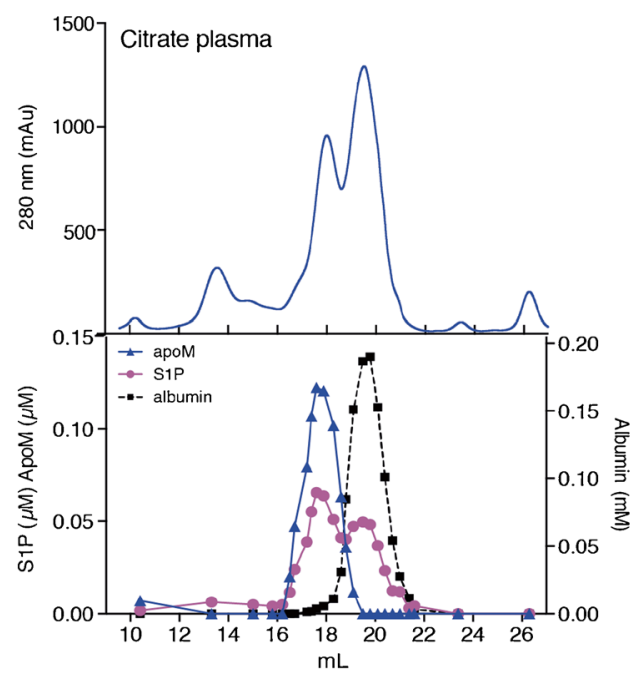

b

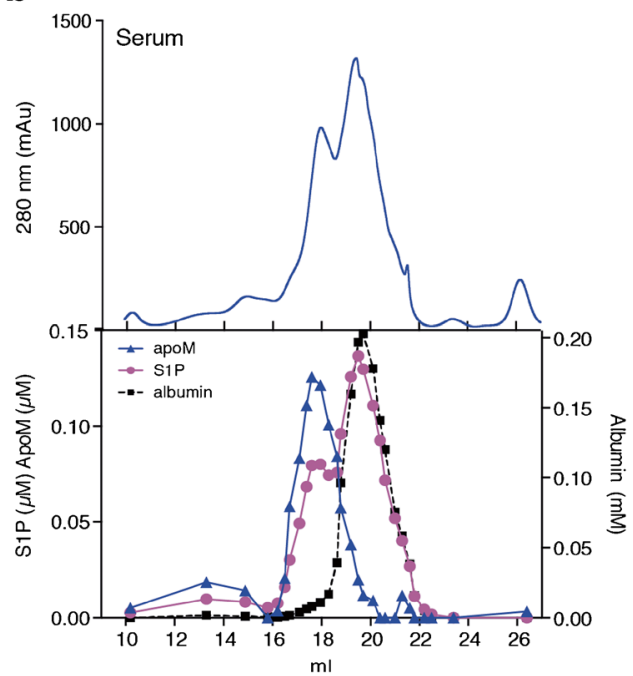

\section{S1P and apoM in different types of plasma and serum}

The effect of different anticoagulants and serum on S1P levels has been evaluated before [12], but the correlation between apoM and S1P has not been assessed. S1P levels in citrate-, Li-hep-, and EDTA-plasma and serum were analysed by collecting samples from 15 healthy individuals. The plasma $\mathrm{S} 1 \mathrm{P}$ concentration ranged between 0.5 and $1.2 \mu \mathrm{M}$, in agreement with previously reported results $[32,23,17]$, whereas the serum S1P levels were between 1.4 and $1.8 \mu \mathrm{M}$, which is higher than those reported before (Fig. 4A) [12]. S1P levels in Li-hep- and EDTA-plasma were slightly higher than that in citrate-plasma (Li-hep $p<0.05)$. Serum S1P levels were significantly higher than S1P levels in citrate- and EDTA-plasma but not significantly different from that in Li-hep-plasma (Fig. 4A). These results were consistent after centrifuging the samples additionally at $20,000 \mathrm{~g}$ for $20 \mathrm{~min}$ (data not shown). ApoM was measured in the same samples and found to be slightly lower in serum and citrate-plasma as compared to EDTA-plasma $(p<0.05)$ (Fig. 4B). ApoM and S1P levels correlated most strongly in EDTA-plasma $(r=0.66$, $p=0.0089)$ and citrate-plasma $(r=0.63, p=0.013)$, whereas there was no significant correlation in Li-hep $(r=0.42$, $p=0.12)$ and serum $(r=0.23, p=0.4)$ (Fig. 4C).

\section{S1P released during blood clotting binds mainly to albumin and not to apoM}

Since the S1P concentration was so much higher in serum than in citrate-plasma, we were interested in studying whether the S1P that is released during coagulation is taken up by apoM or if it binds to albumin. Plasma and serum were applied to gel filtration chromatography and fractions analysed for $\mathrm{S} 1 \mathrm{P}$, apoM and albumin. In citrate-plasma, approximately $60 \%$ of total S1P co-eluted with apoM and $40 \%$ with albumin
(Fig. 5A). However, in serum, opposite results were obtained, with $35 \%$ of total S1P co-eluting with apoM and $65 \%$ with albumin (Fig. 5B).

\section{Discussion}

We present a highly selective LC-MS/MS method for the quantification of S1P, using deuterium-marked S1P as internal standard. The method, which includes a simple sample preparation, has negligible carryover and enhanced performance as compared to previously published methods.

We observed that S1P spiked into pure methanol was unstable, whereas S1P was stable both when spiked in albumincontaining solution and in methanol extracts of serum/plasma. This was likely explained by the presence of apoM and other HDL-proteins in the methanol extract that could serve to stabilise S1P. Presumably, the proteins lose their tertiary structures in the methanol phase, thus increasing the accessibility of S1P in the LC-MS/MS analysis step.

Optimally, the matrix used for S1P calibration would be plasma, where S1P is present normally. However, efforts to remove S1P from plasma with activated charcoal did not result in complete depletion of S1P. Instead, an albumin-based matrix was used, which gave excellent linearity and low background. Recovery analysis yielded 95-111\% recovery of spiked S1P, demonstrating that S1P in plasma could be accurately quantified. Carryover effects of S1P between injections is a known phenomenon $[15,23,22]$. We were able to decrease the carryover to less than $0.07 \%$. This is most likely due to repelling forces between the positively charged surface of the LC column and the positively charged amino group on S1P, which made S1P bind less strongly to the stationary phase. 
Both platelets and erythrocytes store and release S1P $[33,7,9,10,32]$. In a contracted blood clot, erythrocytes adopt a polyhedral shape in a densely packed tessellated configuration with platelets and fibrin lining the exterior [34]. Similar polyhedral erythrocyte structures also arise when anticoagulated blood is centrifuged at $\geq 1000 \mathrm{~g}$ [34]. This extensive cellular re-arrangement may affect S1P release from the erythrocytes and possibly explains the higher S1P we noticed in plasma samples that had been subjected to the high-speed centrifugations. However, sufficient centrifugal forces are needed to ensure proper removal of platelets, as platelet contamination increases the measured S1P. Centrifugation of citrate-plasma at $1000 \mathrm{~g}$ for at least $10 \mathrm{~min}$ was required to remove $\geq 90 \%$ of the platelets. Since S1P slightly increased and apoM slightly decreased upon increasing the centrifugation speed and time, the best correlation between the two parameters was seen after centrifugation of plasma at $1000 \mathrm{~g}$ for $10 \mathrm{~min}$ or $2000 \mathrm{~g}$ for $10 \mathrm{~min}$, which are suitable standard protocols for analysis of apoM and S1P plasma samples. In addition, the correlation between S1P and apoM was strongest in EDTA- and citrate-plasma, which should therefore preferentially be chosen for apoM and S1P analysis in biological samples.

By comparing S1P elution profiles on gel filtration chromatography of citrate-plasma and serum, we observed that most S1P released during blood clotting was preferentially bound to albumin rather than to apoM in HDL. This is consistent with a report showing that platelets release more S1P in the presence of albumin than in the presence of lipoproteins [4]. However, whether this is due to saturation of apoM or the requirement of a specific uptake mechanism for S1P entry into apoM is unknown. Since S1P bound to HDL has been suggested to have different functions than S1P bound to albumin [35], it is possible that S1P released during blood clotting has a distinct biological function from S1P circulating in HDL. S1P have several functions in coagulation $[1,36]$. However, the role of the different plasma pools of S1P, i.e. the S1P carried by apoM in HDL and that carried by albumin, in the homeostasis of blood coagulation is yet to be clarified.

In conclusion, we have developed a highly sensitive and specific LC-MS/MS method for measuring S1P in biological samples, providing in addition a standardised sample collection and preparation protocol. As many parameters may affect S1P release into the samples after the actual blood collection, standardised sample handling procedures need to be used to obtain reproducible results when comparing both inter-study variation as well as variations between samples collected from different patient groups.
Conflict of interest The authors declare that they have no competing interests.

Open Access This article is distributed under the terms of the Creative Commons Attribution 4.0 International License (http:// creativecommons.org/licenses/by/4.0/), which permits unrestricted use, distribution, and reproduction in any medium, provided you give appropriate credit to the original author(s) and the source, provide a link to the Creative Commons license, and indicate if changes were made.

\section{References}

1. Obinata H, Hla T (2012) Sphingosine 1-phosphate in coagulation and inflammation. Semin Immunopathol 34(1):73-91. doi:10. 1007/s00281-011-0287-3

2. Kunkel GT, Maceyka M, Milstien S, Spiegel S (2013) Targeting the sphingosine-1-phosphate axis in cancer, inflammation and beyond. Nat Rev Drug Discov 12:688-701

3. Sanchez T, Hla T (2004) Structural and functional characteristics of S1P receptors. J Cell Biochem 92(5):913-922. doi:10.1002/jcb. 20127

4. Aoki S, Yatomi Y, Ohta M, Osada M, Kazama F, Satoh K, Nakahara K, Ozaki Y (2005) Sphingosine 1-phosphate-related metabolism in the blood vessel. J Biochem 138(1):47-55. doi:10. 1093/jb/mvi100

5. Murata N, Sato K, Kon J, Tomura H, Yanagita M, Kuwabara A, Ui M, Okajima F (2000) Interaction of sphingosine 1-phosphate with plasma components, including lipoproteins, regulates the lipid receptor-mediated actions. Biochem J 352(Pt 3):809-815

6. Christoffersen C, Obinata H, Kumaraswamy SB, Galvani S, Ahnstrom J, Sevvana M, Egerer-Sieber C, Muller YA, Hla T, Nielsen LB, Dahlback B (2011) Endothelium-protective sphingosine-1-phosphate provided by HDL-associated apolipoprotein M. Proc Natl Acad Sci U S A 108(23):9613-9618. doi:10. 1073/pnas. 1103187108

7. Jonnalagadda D, Sunkara M, Morris AJ, Whiteheart SW (2014) Granule-mediated release of sphingosine-1-phosphate by activated platelets. Biochim Biophys Acta 1841(11):1581-1589. doi:10. 1016/j.bbalip.2014.08.013

8. Pappu R, Schwab SR, Cornelissen I, Pereira JP, Regard JB, Xu Y, Camerer E, Zheng YW, Huang Y, Cyster JG, Coughlin SR (2007) Promotion of lymphocyte egress into blood and lymph by distinct sources of sphingosine-1-phosphate. Science 316(5822):295-298. doi:10.1126/science.1139221

9. Ito K, Anada Y, Tani M, Ikeda M, Sano T, Kihara A, Igarashi Y (2007) Lack of sphingosine 1-phosphate-degrading enzymes in erythrocytes. Biochem Biophys Res Commun 357(1):212-217. doi:10.1016/j.bbrc.2007.03.123

10. Hanel P, Andreani P, Graler MH (2007) Erythrocytes store and release sphingosine 1-phosphate in blood. FASEB J : Off Publ Fed Am Soc Exp Biol 21(4):1202-1209. doi:10.1096/fj.06$7433 \mathrm{com}$

11. Ceglarek U, Dittrich J, Helmschrodt C, Wagner K, Nofer JR, Thiery J, Becker S (2014) Preanalytical standardization of sphingosine-1phosphate, sphinganine-1-phosphate and sphingosine analysis in human plasma by liquid chromatography-tandem mass spectrometry. Clin Chim Acta; Int J Clin Chem 435:1-6. doi:10.1016/j.cca. 2014.04.010

12. Hammad SM, Pierce JS, Soodavar F, Smith KJ, Al Gadban MM, Rembiesa B, Klein RL, Hannun YA, Bielawski J, Bielawska A (2010) Blood sphingolipidomics in healthy humans: impact of 
sample collection methodology. J Lipid Res 51(10):3074-3087. doi:10.1194/jlr.D008532

13. Bielawski J, Pierce JS, Snider J, Rembiesa B, Szulc ZM, Bielawska A (2009) Comprehensive quantitative analysis of bioactive sphingolipids by high-performance liquid chromatographytandem mass spectrometry. Methods Mol Biol 579:443-467. doi: 10.1007/978-1-60761-322-0 22

14. Caligan TB, Peters K, Ou J, Wang E, Saba J, Merrill AH Jr (2000) A high-performance liquid chromatographic method to measure sphingosine 1-phosphate and related compounds from sphingosine kinase assays and other biological samples. Anal Biochem 281(1): 36-44. doi:10.1006/abio.2000.4555

15. Berdyshev EV, Gorshkova IA, Garcia JG, Natarajan V, Hubbard WC (2005) Quantitative analysis of sphingoid base-1-phosphates as bisacetylated derivatives by liquid chromatography-tandem mass spectrometry. Anal Biochem 339(1):129-136. doi:10.1016/j.ab. 2004.12.006

16. He X, Huang CL, Schuchman EH (2009) Quantitative analysis of sphingosine-1-phosphate by HPLC after napthalene-2,3dicarboxaldehyde (NDA) derivatization. J Chromatogr B Anal Technol Biomed Life Sci 877(10):983-990. doi:10.1016/j. jchromb.2009.02.048

17. Scherer M, Schmitz G, Liebisch G (2009) High-throughput analysis of sphingosine 1-phosphate, sphinganine 1-phosphate, and lysophosphatidic acid in plasma samples by liquid chromatography-tandem mass spectrometry. Clin Chem 55(6): 1218-1222. doi:10.1373/clinchem.2008.113779

18. Cutignano A, Chiuminatto U, Petruzziello F, Vella FM, Fontana A (2010) UPLC-MS/MS method for analysis of sphingosine 1phosphate in biological samples. Prostaglandins Other Lipid Mediat 93(1-2):25-29. doi:10.1016/j.prostaglandins.2010.06.001

19. Yatomi Y, Ruan F, Ohta J, Welch RJ, Hakomori S, Igarashi Y (1995) Quantitative measurement of sphingosine 1-phosphate in biological samples by acylation with radioactive acetic anhydride. Anal Biochem 230(2):315-320

20. Bielawski J, Szulc ZM, Hannun YA, Bielawska A (2006) Simultaneous quantitative analysis of bioactive sphingolipids by high-performance liquid chromatography-tandem mass spectrometry. Methods 39(2):82-91. doi:10.1016/j.ymeth.2006.05.004

21. Edsall LC, Spiegel S (1999) Enzymatic measurement of sphingosine 1-phosphate. Anal Biochem 272(1):80-86. doi:10.1006/abio. 1999.4157

22. Bode C, Graler MH (2012) Quantification of sphingosine-1phosphate and related sphingolipids by liquid chromatography coupled to tandem mass spectrometry. Methods Mol Biol 874: 33-44. doi:10.1007/978-1-61779-800-9 3

23. Karuna R, Park R, Othman A, Holleboom AG, Motazacker MM, Sutter I, Kuivenhoven JA, Rohrer L, Matile H, Hornemann T, Stoffel M, Rentsch KM, von Eckardstein A (2011) Plasma levels of sphingosine-1-phosphate and apolipoprotein $\mathrm{M}$ in patients with monogenic disorders of HDL metabolism. Atherosclerosis 219(2): 855-863. doi:10.1016/j.atherosclerosis.2011.08.049

24. Schmidt H, Schmidt R, Geisslinger G (2006) LC-MS/MS-analysis of sphingosine-1-phosphate and related compounds in plasma samples. Prostaglandins Other Lipid Mediat 81(3-4):162-170. doi:10. 1016/j.prostaglandins.2006.09.003
25. Lan T, Bi H, Liu W, Xie X, Xu S, Huang H (2011) Simultaneous determination of sphingosine and sphingosine 1-phosphate in biological samples by liquid chromatography-tandem mass spectrometry. J Chromatogr B Anal Technol Biomed Life Sci 879(7-8):520 526. doi:10.1016/j.jchromb.2011.01.015

26. Saigusa D, Shiba K, Inoue A, Hama K, Okutani M, Iida N, Saito M, Suzuki K, Kaneko T, Suzuki N, Yamaguchi H, Mano N, Goto J, Hishinuma T, Aoki J, Tomioka Y (2012) Simultaneous quantitation of sphingoid bases and their phosphates in biological samples by liquid chromatography/electrospray ionization tandem mass spectrometry. Anal Bioanal Chem 403(7):1897-1905. doi:10.1007/ s00216-012-6004-9

27. Ruwisch L, Schafer-Korting M, Kleuser B (2001) An improved high-performance liquid chromatographic method for the determination of sphingosine-1-phosphate in complex biological materials. Naunyn Schmiedeberg's Arch Pharmacol 363(3):358-363

28. Vogeser M, Parhofer KG (2007) Liquid chromatography tandemmass spectrometry (LC-MS/MS) - technique and applications in endocrinology. Exp Clin Endocrinol Diabetes : Off J German Soc Endocrinol German Diabetes Assoc 115(9):559-570. doi:10.1055/ s-2007-981458

29. Axler O, Ahnström J, Dahlbäck B (2007) An ELISA for apolipoprotein $\mathrm{M}$ reveals a strong correlation to total cholesterol in human plasma. J Lipid Res 48(8):1772-1780. doi:10.1194/jlr.M700113JLR200

30. Christoffersen C, Nielsen LB, Axler O, Andersson A, Johnsen AH, Dahlback B (2006) Isolation and characterization of human apolipoprotein M-containing lipoproteins. J Lipid Res 47(8):1833-1843. doi:10.1194/jlr.M600055-JLR200

31. Axler O, Ahnstrom J, Dahlback B (2008) Apolipoprotein M associates to lipoproteins through its retained signal peptide. FEBS Lett 582(5):826-828. doi:10.1016/j.febslet.2008.02.007

32. Bode C, Sensken SC, Peest U, Beutel G, Thol F, Levkau B, Li Z, Bittman R, Huang T, Tolle M, van der Giet M, Graler MH (2010) Erythrocytes serve as a reservoir for cellular and extracellular sphingosine 1-phosphate. J Cell Biochem 109(6):1232-1243. doi:10. 1002/jcb.22507

33. Yatomi Y, Yamamura S, Ruan F, Igarashi Y (1997) Sphingosine 1phosphate induces platelet activation through an extracellular action and shares a platelet surface receptor with lysophosphatidic acid. J Biol Chem 272(8):5291-5297

34. Cines DB, Lebedeva T, Nagaswami C, Hayes V, Massefski W, Litvinov RI, Rauova L, Lowery TJ, Weisel JW (2014) Clot contraction: compression of erythrocytes into tightly packed polyhedra and redistribution of platelets and fibrin. Blood 123(10):15961603. doi:10.1182/blood-2013-08-523860

35. Wilkerson BA, Grass GD, Wing SB, Argraves WS, Argraves KM (2012) Sphingosine 1-phosphate (S1P) carrier-dependent regulation of endothelial barrier: high density lipoprotein (HDL)-S1P prolongs endothelial barrier enhancement as compared with albuminS1P via effects on levels, trafficking, and signaling of S1P1. J Biol Chem 287(53):44645-44653. doi:10.1074/jbc.M112.423426

36. Rauch BH (2014) Sphingosine 1-phosphate as a link between blood coagulation and inflammation. Cell Physiol Biochem 34(1):185196. doi: $10.1159 / 000362994$ 